\title{
Reviews
}

\section{Psychology in the Bathroom}

By Nick Haslam

Palgrave Macmillan, 2012, £50.00, hb, 184 pp.

ISBN: 9780230368248

With its centrality to psychoanalytic thought, excretion was once essential to the understanding of human motivation and personality. No longer, writes Professor Nick Haslam, author of Psychology in the Bathroom. Perhaps embarrassed by fanciful past theories, excretion is all but ignored by modern psychology. Psychology journals on eating disorders abound, Haslam writes, but there is not a single title devoted to the terminal end of the digestive system.

Haslam aims to redress this balance, and presents an unembarrassed survey of the psychology of excretion: flatulence, diarrhoea, constipation, urinary incontinence, inhibition and perversion - all are here. The birth of the 'anal character' and its re-emergence as obsessive-compulsive personality disorder is also discussed, as are toilet graffiti and swearing.

The tone is academic rather than journalistic, but the book is nevertheless readable and engaging. An examination of irritable bowel syndrome offers insight into the intimacy of mind and viscera. Flatulence is shown to have connections to many forms of psychopathology. The anxious, for instance, may suffer from an excess of flatus because of hyperventilation. Coprophagia might disgust, but Haslam relates that it can reveal disorders as varied as dementia, psychosis and obsessive-compulsive disorder

Memorable are the book's numerous lively vignettes. Researchers investigated urinary hesitancy in public conveniences with the aid of a stopwatch, a mirror and ears primed for the sound of urine hitting porcelain. Psychoanalysts put the concept of the anal character to such extensive use that even seemingly benign children's stories received their attention. It is Goldilock's bottom, after all, which breaks the bear's chair. Toilet graffiti may be in decline as this discourse has now moved online.

I do recommend this book to psychiatrists everywhere, but I also wonder whom Haslam primarily has in mind as a reader. I doubt he has the popular science market in his sights as this book assumes a fair amount of prior knowledge on the part of the reader and is priced for academia. Yet can a book that devotes an entire chapter to toilet seat behaviour ('Up or down?' - the answer is actually quite complicated) lay claim to being entirely serious?

Stephen Ginn, ST5 General Adult Psychiatry, East London NHS Foundation Trust, South Hackney Community Mental Health Team, Donald Winnicott Centre, London, UK, email: mail@stephenginn.com

doi: $10.1192 / p b . b p .112 .041426$

\section{The Psychedelic Renaissance: Reassessing the Role of Psychedelic Drugs in 21st Century Psychiatry and Society}

\section{Ben Sessa}

Muswell Hill Press, 2012, E16.95, pb, 240 pp. ISBN: 9781908995001

Throughout the period of training and in clinical practice, there is a tendency to develop a prejudiced view about 'illicit' or 'illegal' street drugs, including psychedelics. Many clinicians hold the simple view that cannabis exacerbates psychosis and heroin dependence is treated with substitute prescribing, and have little exposure to patients who misuse psychedelics. This in itself is a clue to understanding the essence of this book, namely that although psychedelic use is more prevalent than either cannabis or heroin use, this is not reflected in clinical practice, nor is it reflected in medical education.

In this well-researched and well-referenced book, the author has in a very logical manner explained that he is not arguing for the legalisation of psychedelic street drugs. Rather, he champions the cause of raising awareness and generating interest for further research into the medical use of psychedelics, predominantly as a tool to enhance psychotherapy interventions, but also drawing attention to their potential for treating other psychiatric conditions.

Dr Sessa's reflections on how he became interested in psychedelic drug research make for an interesting read. $\mathrm{He}$ classifies and describes the effects of psychedelics in a minitextbook fashion. His interest and expertise in this field are evident. What I found particularly fascinating was the hypothesis of how naturally occurring psychedelics may have influenced the evolution of culture and religion, and the author goes to great length in providing examples, through religious texts, of the use of these substances in the ancient world. He best sums it up when he concludes, 'Psychiatry needs psychedelics, and psychedelics need psychiatry'. I couldn't agree more.

Sarabjeet Kohli specialty registrar ST6 in substance misuse psychiatry, The Bridge, Birmingham, UK, email: sarabjeet.kohli2@bsmhft.nhs.uk doi: $10.1192 /$ pb.bp.112.042275 